\title{
Large Area Nondestructive Evaluation of a Fatigue Loaded Composite Structure
}

\author{
Mr. Joseph N. Zalameda ${ }^{1}$, Mr. Eric R. Burke ${ }^{1}$, Dr. Michael R. Horne ${ }^{2}$, and Dr. Eric I. Madaras ${ }^{1}$ \\ ${ }^{1}$ NASA Langley Research Center, MS231 Hampton, VA 23681 \\ ${ }^{2}$ National Institute of Aerospace, NASA Langley Research Center, MS231 Hampton, VA 23681 \\ E-mail: joseph.n.zalameda@nasa.gov
}

\begin{abstract}
Large area nondestructive evaluation (NDE) inspections are required for fatigue testing of composite structures to track damage initiation and growth. Of particular interest is the progression of damage leading to ultimate failure to validate damage progression models. In this work, passive thermography and acoustic emission NDE were used to track damage growth up to failure of a composite three-stringer panel. Fourteen acoustic emission sensors were placed on the composite panel. The signals from the array were acquired simultaneously and allowed for acoustic emission location. In addition, real time thermal data of the composite structure were acquired during loading. Details are presented on the mapping of the acoustic emission locations directly onto the thermal imagery to confirm areas of damage growth leading to ultimate failure. This required synchronizing the acoustic emission and thermal data with the applied loading. In addition, processing of the thermal imagery which included contrast enhancement, removal of optical barrel distortion and correction of angular rotation before mapping the acoustic event locations are discussed.
\end{abstract}

Keywords: large area composite inspection, thermography, acoustic emission, fiber break, delamination

\section{INTRODUCTION}

The purpose of this paper is to describe the methodology of measuring damage onset and growth in a composite structure during fatigue loading. Thermal and acoustic emission nondestructive evaluation (NDE) techniques were used to track the damage in real time. When damage growth was detected, the loading was stopped for a more detailed inspection using non-immersion ultrasound. The non-immersion ultrasound was used to provide a detailed assessment of the damage growth through-the-thickness and thus ultimately provided a better understanding of damage progression modes that can lead to ultimate failure [1]. NDE inspections are necessary to provide structural engineers a tool to incrementally control and document damage growth as a function of fatigue cycles before failure. This allows for the comparison of NDE results to develop and validate progressive damage analysis (PDA) models [2-5]. The ultimate goal is to use the validated PDA models to decrease the time required to certify composite structures and therefore save development costs.

Real time NDE can document the progression of damage in between the ultrasonic measurements and also provide the documentation of ultimate failure mechanisms. The technologies used for real time NDE were thermography and acoustic emission. In the past there have been numerous studies combining thermography with acoustic emission [6-10]. Our technique is different where the acoustic emission data were directly mapped onto the thermal imagery. In addition, image rotation and removal of optical distortions on the thermal images were required to accurately map the acoustic emission data onto the thermal images. Mapping the acoustic emission events onto the thermal images provided confirmation of damage growth at areas of heating (due to fiber breaks, rubbing of disbond areas and matrix cracks) and where ultimate failure will occur. This provided a measurement capability to detect growing damage (location and size) for improved structures testing. Multiple infrared (IR) cameras and multiple acoustic emission sensors were employed for full coverage of the structure during testing.

\section{SAMPLE}

The hat stiffened composite panel flat side and stringer side with acoustic emission locations (stringer side) are shown in Figures 1a and $1 \mathrm{~b}$ respectively. The panel skin is 17 plies with a thickness of $0.32 \mathrm{~cm}$. The hat flange is 11 plies with a thickness of $0.21 \mathrm{~cm}$. Barely visible impact damage (BVID) were induced at 4 locations (I1, M1, I2, M2) on the panel skin on the hat stiffeners flange or right next to the flange. The BVID was used to simulate existing delamination damage and to serve as potential damage growth sites. Cyclic compressive loads were applied from $-20,000$ pounds up to $-150,000$ pounds at $2 \mathrm{~Hz}$ using the setup shown in Figure 2. The sample was painted with a speckle pattern for high speed digital image correlation imaging. The high speed digital image correlation cameras required illumination using red light emitting diode (LED) lights. The LED lights were not a problem for the IR cameras operated in the mid IR waveband. The load was 
applied from the bottom while the top was held stationary. Passive thermography was used to track the damage on both the flat and stringer sides during loading using two IR cameras. Shown in Figure $1 \mathrm{~b}$ are the locations of the acoustic emission sensors in relation to the BVID. The acoustic emission sensors were used for real time detection of acoustic events indicating damage growth.

\section{INSPECTION SYSTEMS}

The thermal measurement setups are shown in Figure 3. The basic system consists of two IR cameras operating in the 3-5 micrometer (midwave) IR band and an image data acquisition computer. The IR cameras were both configured with $25 \mathrm{~mm}$ germanium optics. The focal plane array sizes for the cameras were $640 \times 512$ and $1024 \times 1204$. The passive inspection

(a) Flat Side

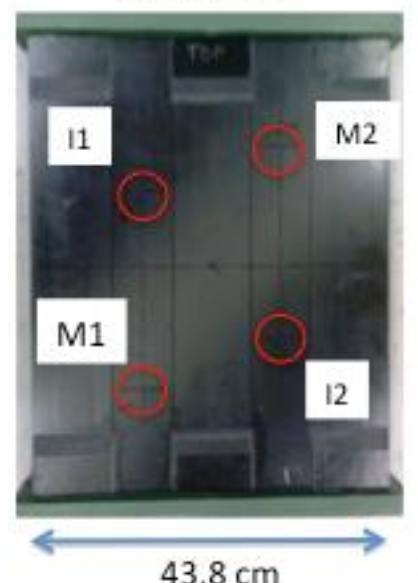

(b) Stringer Side

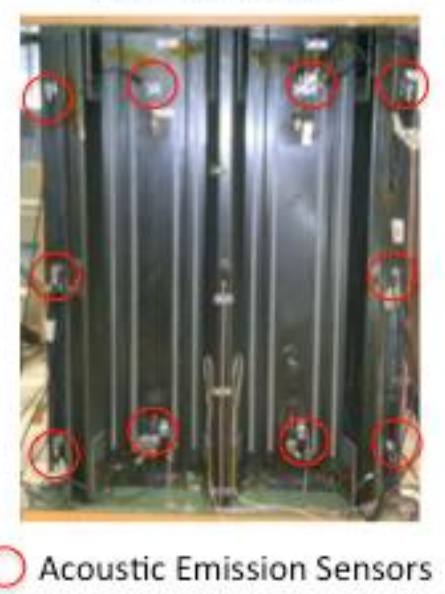

Figure 1: Three stringer composite panel tested with impact and acoustic emission sensor locations.

captured the thermal variations during the fatigue loading. The setup required a Plexiglas ${ }^{\circledR}$ shield to contain fragments if ultimate failure occurred. The IR cameras were located behind the shield with the lens positioned in front of a viewing hole. The added benefit of the shield is it filters out spurious IR background sources. The cameras were synchronized using the load signal from the test machine. The sinusoidal load signal was offset corrected and the zero crossover points were used to produce a TTL trigger signal. The trigger signal was converted to a series of pulses using a signal generator. The IR cameras were operated at a frequency range of 16 to $90 \mathrm{~Hz}$ depending on acquisition time duration. Higher frequencies were used to capture the damage progression as the panel approached ultimate failure. The thermography data were processed using an image differencing technique [6]. Ultrasonic inspections were implemented at intervals determined by the indication of damage growth from thermography and acoustic emission. The ultrasonic inspection technique utilizes a broadband $10 \mathrm{MHz}$ transducer $(1.27 \mathrm{~cm}$ diameter with $5.08 \mathrm{~cm}$ focus) contained in a captive water column with a flexible membrane tip design [1]. Water is misted onto the surface for ultrasonic coupling. The probe is scanned over the entire panel on the flat side. A picture of the setup is shown in Figure 4.

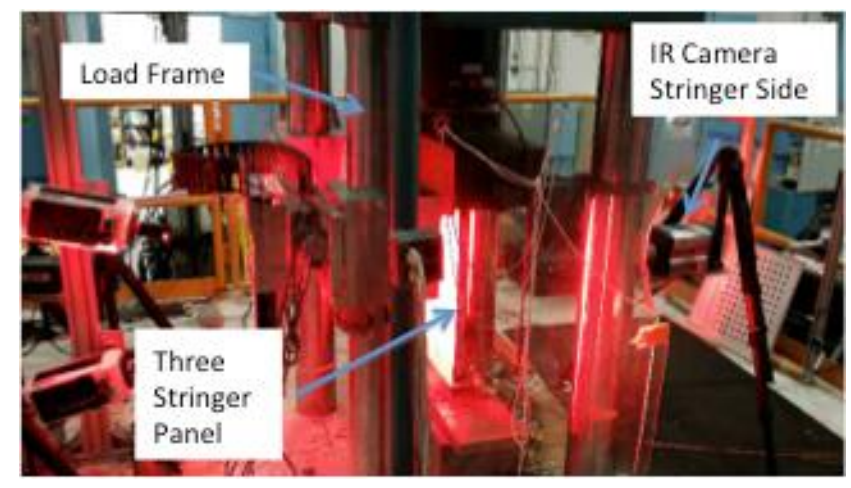

Figure 2: Fatigue loading setup for three-stringer panel. 


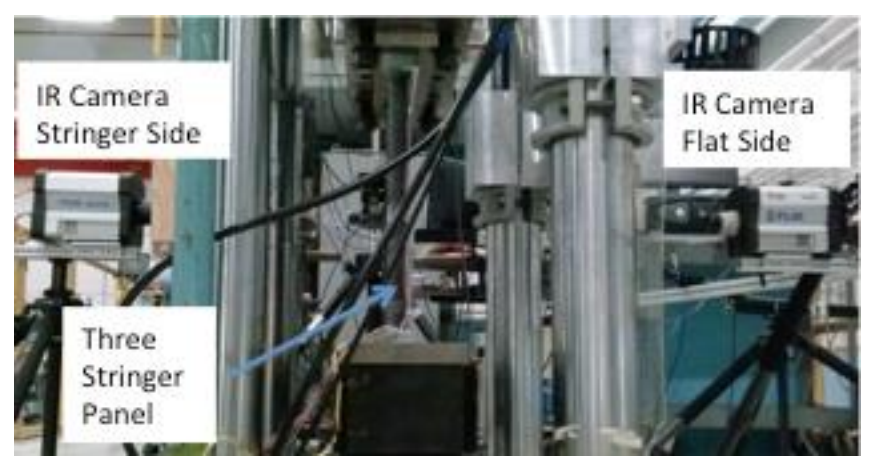

Figure 3: Three-stringer panel setup for passive thermography inspection of both flat and stringer sides.

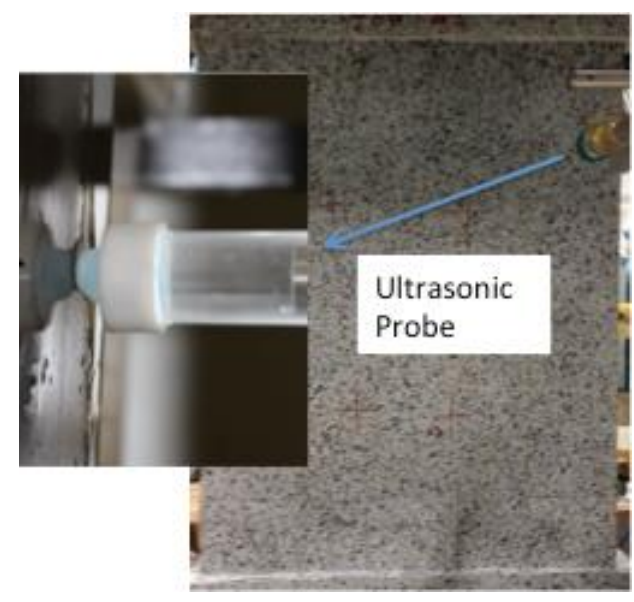

Figure 4: Close up of ultrasonic probe.

Fourteen acoustic emission sensors were bonded to the composite panel on the stringer side (shown in Figure 1b). They were connected to a data acquisition computer via Digital Wave ${ }^{\mathrm{TM}}$ PA0 preamp/line drivers and a Digital Wave ${ }^{\mathrm{TM}}$ FM1 signal conditioning 16 channel amplifier for capture of acoustic data. The acoustic emission computer also captured the load signal for synchronization. Acoustic emission systems collect structure-borne sound, typically not audible, in the ultrasonic frequency band (approx. 50 to $500 \mathrm{kHz}$ ), generated by dynamic displacements such as damage initiation and growth at picometer scales [11]. Pre-cursors to failure are monitored in real-time. The total signal energy, $S E$, of an acoustic signal is given as:

$$
S E=\sum_{i=1}^{n} V_{i}^{2} \Delta t
$$

where $V_{i}$ is the signal voltage, $i$ is the time reference point, $n$ is the number of time points in the signal, $\Delta \mathrm{t}$ is the sampling time per point. Trends of the signal energy over time, or load, are used to identify and track damage development (matrix cracks, fiber breaks, delamination formation). Signals from the distributed array of sensors are acquired simultaneously when any one sensor threshold triggers. This allows calculating the source or event location [11,12].

\section{MEASUREMENT RESULTS}

Examples of processed thermal images of the flat side are shown in Figure 5. The processing technique used to improve defect contrast involves a delayed image subtraction $[6,13]$. A moving buffer is required in the acquisition software in order for this processing technique to be implemented in real time. The delayed subtraction is given as:

$$
\text { Difference Image = Acquired Image }[i]-\text { Previous Image }[i-k / 2]
$$

where $k=$ number of images per cycle and $i$ is the current acquired image number. Dividing $k$ by 2 insures the maximum and minimum temperature images are subtracted within a cycle. This provides a series of images with optimal defect contrast varying with damage depth. Improved defect signal to noise can be obtained by averaging the difference images over a number of cycles if desired. Also shown in Figure 5 are the respective ultrasonic images obtained at intervals determined by the indication of damage growth from thermography and acoustic emission. The acoustic emission events are shown in 
Figures $6 \mathrm{a}$ and $6 \mathrm{~b}$ where the acoustic emission events are shown for different fatigue tests. The accumulated acoustic events for Fig. 6a were for approximately 50 seconds and for Figure $6 \mathrm{~b}$ were for 16.5 seconds. As the structure nears failure the accumulated acoustic events increases as a function of time as shown in Figures $6 \mathrm{a}$ and $6 \mathrm{~b}$.

\section{MAPPING ACOUSTIC EMISSION EVENTS ONTO THERMAL IMAGERY}

An image processing routine was developed to map the acoustic emission data onto the thermal imagery. The procedure is shown in Figure 7 along with some example thermal images. This method required improving image contrast, removing angular rotation, and correcting for optical barrel distortion. In addition, the resolution per pixel was determined

$97.3 \%$ of Life

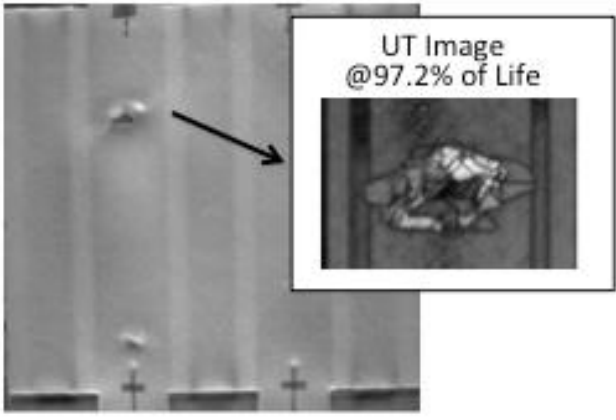

$99.2 \%$ of Life

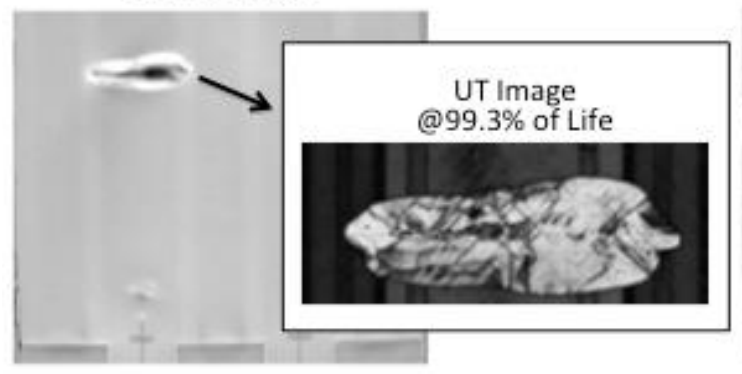

$98.6 \%$ of Life

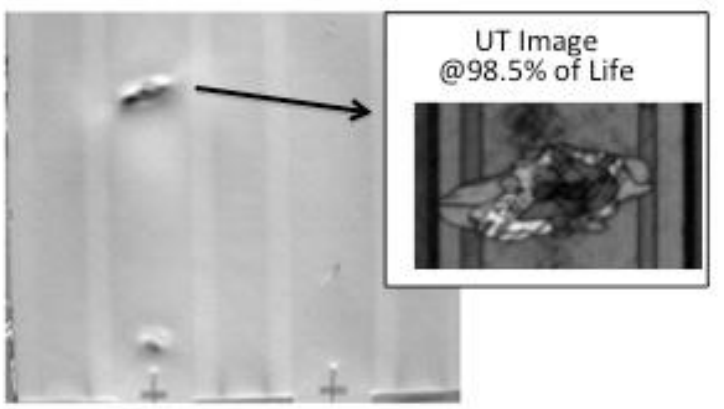

$99.998 \%$ of Life

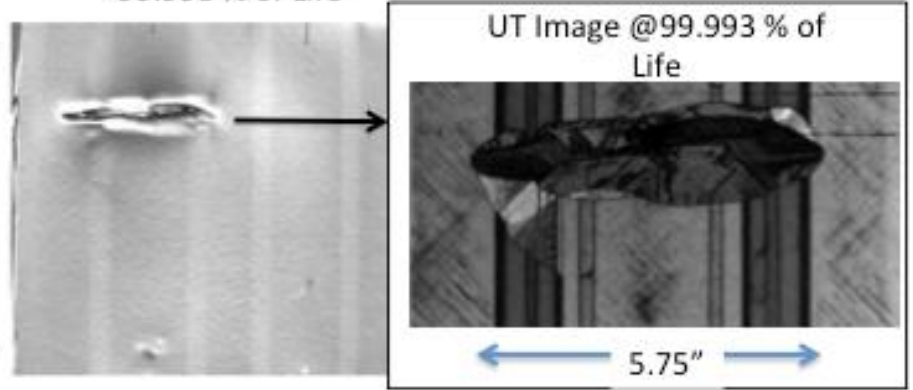

Figure 5: Comparison of processed thermography images to ultrasonic inspection results.

(a) Flat Side of Panel $97.3 \%$ of Life

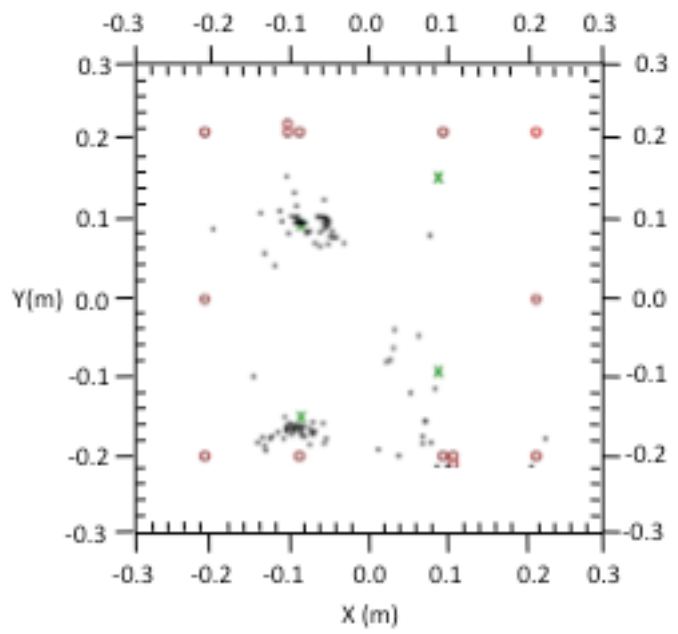

(b) Stringer Side of Panel $99.9 \%$ of Life

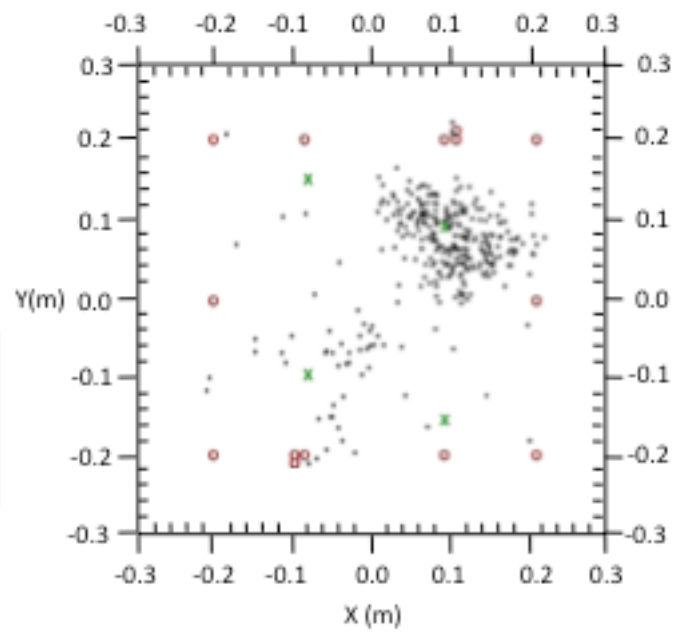

Figure 6: Three-stringer panel acoustic emission event locations. 
by measuring known distances on the composite structure. The acoustic emission data were then mapped onto thermal imagery, revealing the cluster of acoustic emission event locations around the thermal signatures of interest. The removal of angular rotation is performed using an affine transformation [14]. The barrel distortion is corrected using an image forward transformation given by the equation below [15].

$$
r_{\text {undistorted }}=r_{\text {distorted }}\left(1-d\left(r_{\text {distorted }}\right)^{2}\right)
$$

Where $r_{\text {undistorted }}$ is the corrected Cartesian coordinate vector distance from the center of the image and $r$ distorted is the distorted Cartesian vector distance from the center of the image. The parameter $d$ was set to 0.2 to remove the distortion. The pixel resolution was calculated to be 8.2 pixels per $\mathrm{cm}$. A comparison of the contrast enhanced, rotation corrected and barrel distortion thermal images are shown in Figures $7 \mathrm{~b}$ and $7 \mathrm{c}$. The image in Figure $7 \mathrm{c}$ is used to map the acoustic emission data.

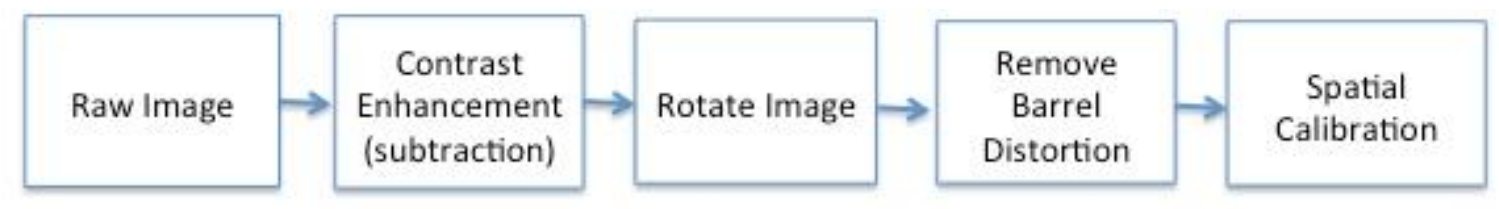

(a) Raw Image

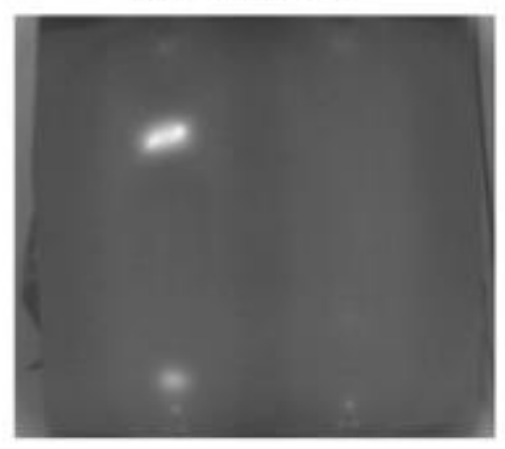

(b) Contrast Enhanced

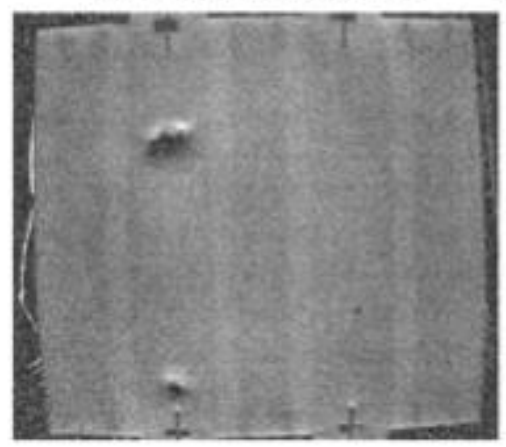

(c) Rotated/Barrel Distortion Removed

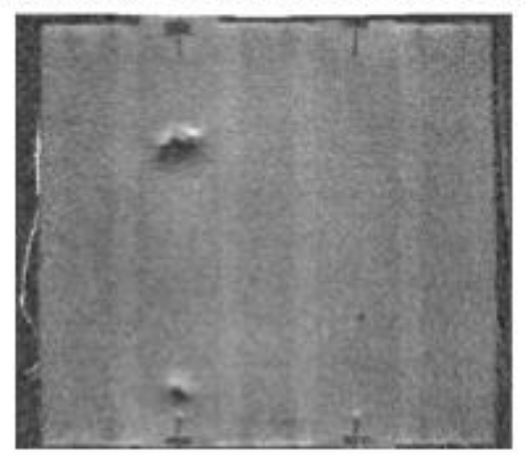

Figure 7. Procedure to process thermal imagery for mapping of acoustic emission data.

The mapped acoustic emission data as a function of remaining life are shown in Figures 8 and 9 for the flat side and stringer side respectively. The impact locations $(\mathrm{X})$ are also shown. Both the thermal data and the acoustic emission data were synchronized to the load and therefore the timing of the acoustic emission events are matched to the thermal image temporally. For a given location, the mapped indicator (star graphic) size and color are based on the event energy given by:

$$
\text { Indicator }=\text { Round }\left[(\text { event energy })^{1 / 4}+10\right]
$$

where the event indicator values of less than 13 (with a minimum size of 10) were given a low energy color of blue (opacity of 0.5 ), indicator values between 13 and 15 were designated as a mid energy event color yellow (opacity of 0.7), and greater than 15 was a high energy event with color red (opacity of 1.0). As the structure nears failure, the accumulated acoustic events and indicator values increase as shown in Figure 8 and 9. Ultimate failure occurred at the location where the red high energy indicators and mostly yellow indicators are clustered. It is important to note the location of the acoustic emission events are prone to errors due to the progression of damage (fiber breaks, delaminations, matrix cracking) as the structure nears failure. These defects can affect the propagation of the acoustic emission signal in terms velocity and waveform mode conversions resulting in processing errors; however, overall the clustered locations are comparable to the thermal indications of the growing damage areas. In particular for the left image (thermal image from the stringer side) in Figure 9, some of the clustered points tend to accumulate below the delamination on the right side. This clustering was due to a growing disbond between the stringer flange and the skin below the delamination. This disbond between the stringer flange and skin caused ultimate failure.

\section{CONCLUSIONS}

By combining thermography with acoustic emission a large area, real time NDE inspection technique was developed. This technique allows for the determination of damage growth areas and failure location of a loaded composite structure to be accurately determined. A particular area of heating, in addition to confirmation of clustered acoustic emission 

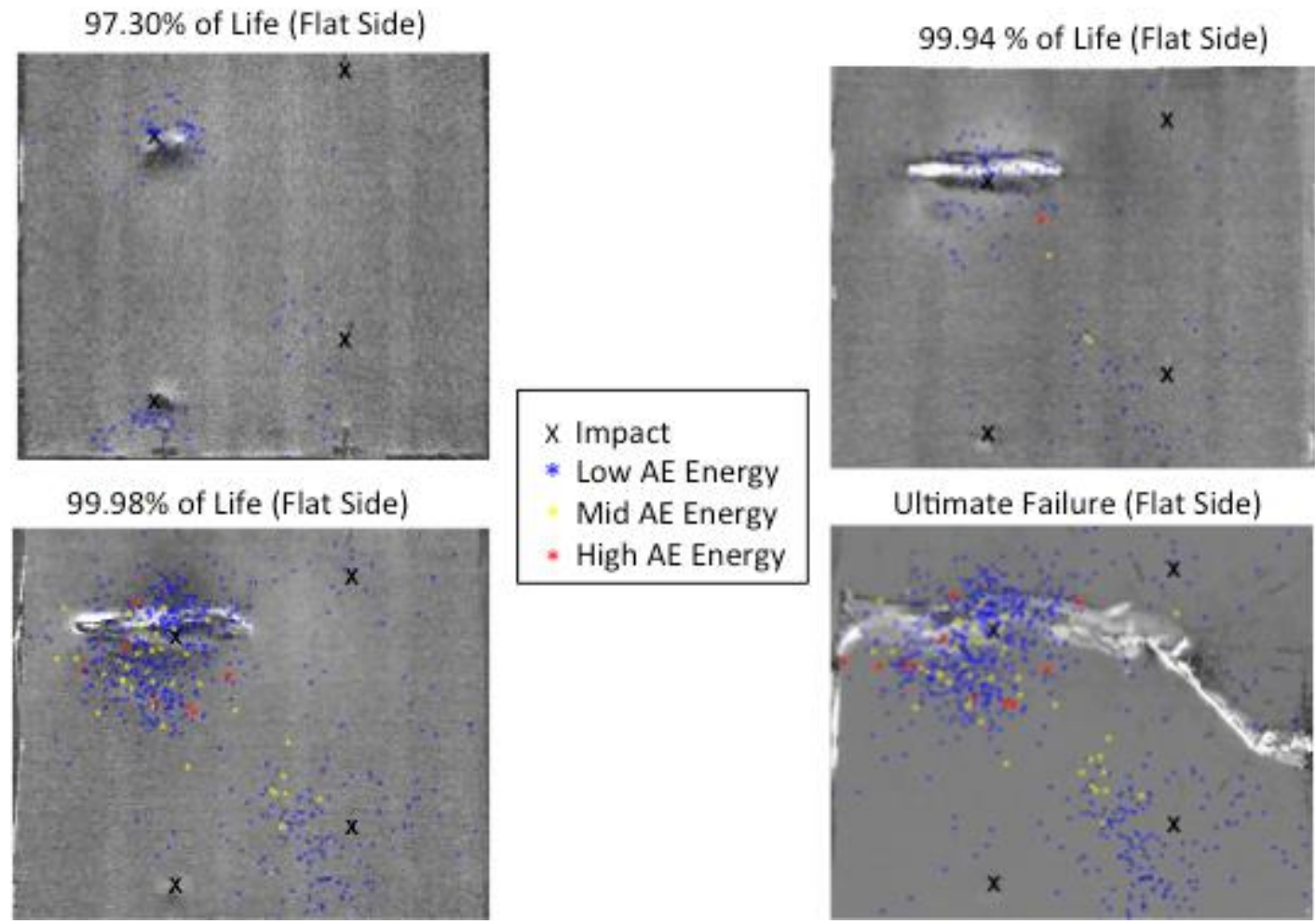

Figure 8. Mapping of acoustic emission data onto processed thermal imagery (Flat Side).

99.94\% of Life (Stringer Side)

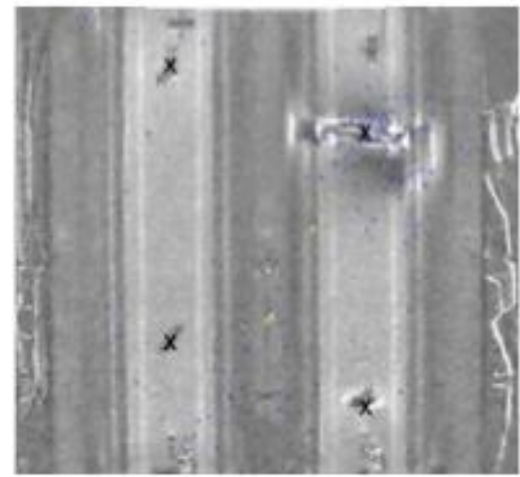

$99.98 \%$ of Life (Stringer Side)

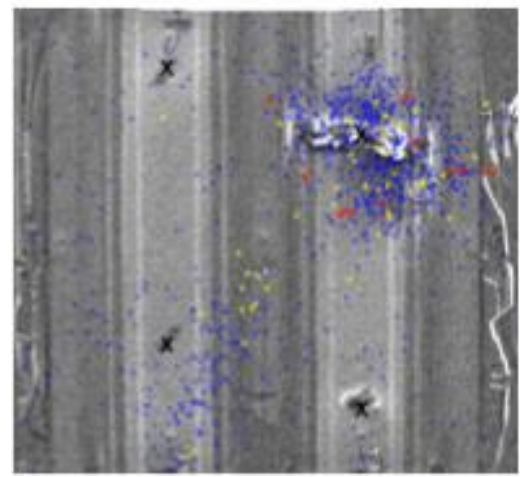

99.95\% of Life (Stringer Side)

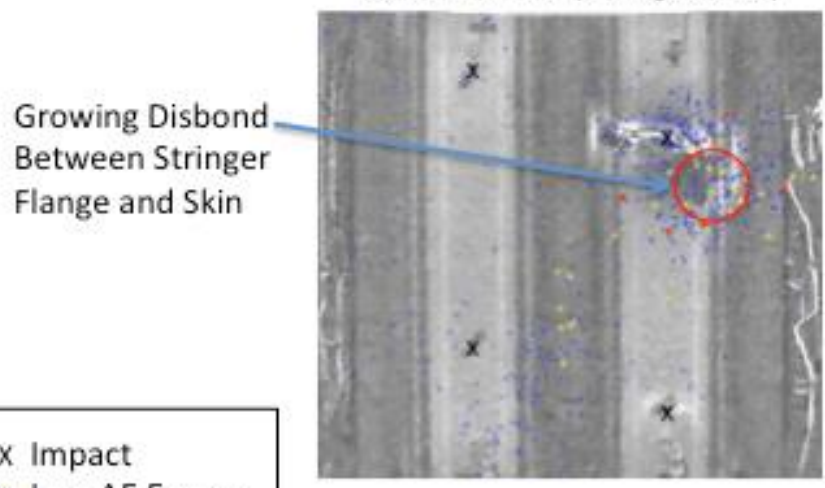

* Low AE Energy Mid AE Energy

* High AE Energy

Figure 9. Mapping of acoustic emission data onto processed thermal imagery (Stringer Side). 
events, were a good indicator of damage growth and where ultimate failure occurred. An image processing procedure has been presented to allow for mapping of the acoustic emission events onto the thermal images. This technique has shown potential for real time inspection of large area aerospace structures during load testing.

\section{ACKNOWLEDGEMENTS}

The authors would like to acknowledge Mr. Wade Jackson of NASA Langley for ultrasonic inspections, sample preparation, and mounting. Also the authors would like to acknowledge Mr. William Johnston of Lockheed Martin for testing support and Mr. Kenneth McLarney, NASA Internship, Fellowship, and Scholarships (NIFS) student for data processing.

\section{REFERENCES}

[1] Johnston P. H., Wright C. W., Zalameda J. N., and Seebo J. P., "Ultrasonic monitoring of ply crack and delamination formation in composite tube under torsion load", Ultrasonic Symposium (IUS), IEEE, pp. 595 - 598, (2010).

[2] O'Brien, T. K., "Development of a Delamination Fatigue Methodology for Composite Rotorcraft Structure", NASA Aviation Safety Technical Conference Denver, CO, (October 2008).

[3] Krueger, R., "An Approach to Assess Delamination Propagation Simulation Capabilities in Commercial Finite Element Codes", NASA/TM-2008-215123, 2008.

[4] Davis, A., R., Bush, C., Harvey, J. C. and Foley, M. F., "Fresnel lenses in rear projection displays," SID Int. Symposium Digest Tech. Papers 32(1), 934-937, (2001).

[5] Bisagni, C., Dávila, C. G., Rose, C., and Zalameda, J. N., "Experimental Evaluation of Damage Progression in Postbuckled Single Stiffener Composite Specimens", American Society for Composites $29^{\text {th }}$ Technical Conference Proceedings, US-Japan 16, ASTM D30, (Feb. 2014).

[6] Zalameda, J. N., Burke, E. R., Parker, R. F., Seebo, J. P., Wright, C. W., and Bly, J. B., "Thermography Inspection for Early Detection of Composite Damage in Structures during Fatigue Loading", Thermosense XXXIV, edited by Douglas Burleigh, Gregory R. Stockton, Proc. of SPIE Vol. 835403 (April 2012).

[7] La Rosa, G., Clienti, T., Savio, F. Lo, "Fatigue Analysis by Acoustic Emission and Thermographic Techniques", Procedia Engineering, XVII International Colloquium on Mechanical Fatgue of Metals, (ICMFM17), Volume 74, pp. 261-268, 2014.

[8] Kordatos E., Dassios K., Aggelis D., Matikas T., "Rapid evaluation of the fatigue limit in composites using infrared lock-in thermography and acoustic emission", Mech Res Commun, 54 (2013), pp. 14-20

[9] Munoz, V., Vales, B., Perrin, M., Pastor, M. L., Welemane, H., Cantarel, A., and Karama, M., "Damage detection in CFRP by coupling acoustic emission and infrared thermography", Composites Part B: Engineering, Volume 85, pp. 6875, February 2015.

[10] Ringermacher H. I., et al, "System and Method for Locating Failure Events in Samples Under Load", United States Patent No. 7,516,663 B2, (April 14, 2009).

[11] Horne, M. R. "Rayleigh Wave Acoustic Emission during Crack Propagation in Steel". PhD Dissertation, Virginia Polytechnic Institute and State University. (2003).

[12]Ziola S. M. and Gorman M. R., "Source location in thin plates using cross-correlation", J. of Acoustic Society of America, 90 (5), (1991).

[13] Winfree, W. P., Zalameda, J. N., and Howell, P. A., "Improved flaw detection and characterization with difference thermography", Proc. SPIE 8013, 80130U (April 2011).

[14] Weisstein, Eric W., "Affine Transformation.", From MathWorld-A Wolfram Web Resource. http://mathworld.wolfram.com/AffineTransformation.html. (accessed 5 October 2015).

[15] Gribbon, K. T., C. T. Johnston, and Donald G. Bailey. "A real-time FPGA implementation of a barrel distortion correction algorithm with bilinear interpolation." In Image and Vision Computing New Zealand, pp. 408-413. 2003. 